\title{
THE LATEST FASHIONS IN SKIN DISEASE
}

\author{
Joseph M. Carroll, Ph.D., ${ }^{*}$ and Lowell A. Goldsmith, M.D. ${ }^{\dagger}$ \\ *Keratinocyte Laboratory, Imperial Cancer Research Fund, \\ London, England ${ }^{\dagger}$ Department of Dermatology, \\ University of Rochester, Rochester, New York, U.S.A.
}

The $1.75 \mathrm{~m}^{2}$ epidermis is a major interface between the body and the environment. Though only a few hundred microns in thickness, this organ provides a barrier to physical and chemical trauma through its highly ordered cellular structure and its synthesis of specialized proteins and lipids. Human epidermis is composed primarily of 10-20 cell layers of highly organized keratinocytes attached to a complex basement membrane overlying the dermis (Fig. 1). The basal layer of this tissue is the site of most mitotic activity. As keratinocytes lose adhesion to the basement membrane, they leave the basal layer and begin to undergo a progressive terminal differentiation, culminating in large, anucleated stratum corneum cells with abundant, relatively insoluble intracellular proteins. The epidermal layers can be characterized by different morphological appearances and unique patterns of gene expression. The unique spectrum of gene expression in different layers is mirrored by the existence of skin diseases limited to individual compartments of the epidermis. In the last few years the molecular basis of several of these skin diseases has been elucidated.

\section{DISEASES OF THE KERATINS}

Keratins are the most abundant gene products of keratinocytes. These proteins are $\alpha$-helical and form heterodimeric pairs of acidic and basic partners to form $10 \mathrm{~nm}$ intermediate filaments. Different keratin pairs in the basal and suprabasal layers share the feature of imparting structural integrity. Albers and Fuchs have shown, by transfecting mutated keratins into keratinocytes in vitro, that mutations of the helical rod domain of the proteins are the most disruptive and result in a collapse of the filament network and overall cell fragility (1). The major keratin pairs found in the basal layer of the epidermis are keratins 5 and 14 ( $\mathrm{K} 5$ and $\mathrm{K} 14$ ); keratins 1 and 10 ( $\mathrm{K} 1$ and $\mathrm{K} 10$ ) are the predominant suprabasal keratin pair.

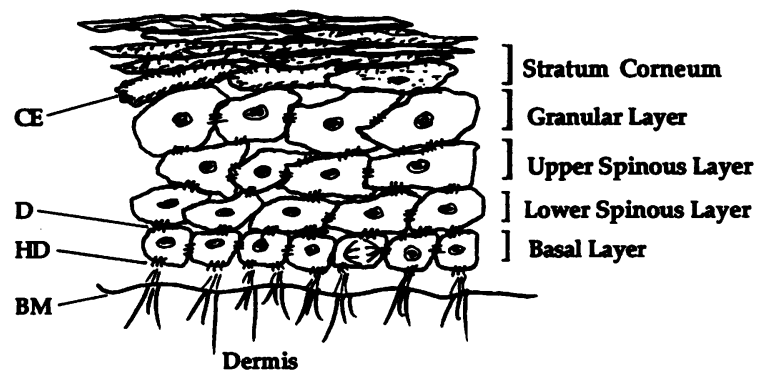

FIG. 1. A schematic representation of the epidermis

The basal layer rests upon a basement membrane (BM) which is composed of extracellular matrix proteins and separates the epidermis from the dermis. Cell division takes place in the basal layer, and as cells leave this layer they undergo a progressive terminal differentiation culminating in enlarged, anucleate cells of the stratum corneum. The major intracellular proteins present in the keratinocytes of all layers are the keratins, which impart structural integrity. The hemi-desmosomes (HD) are structures responsible for connecting the basal cells to the anchoring filaments of the basement membrane, while desmosomes (D) provide cell-cell adhesion in suprabasal layers. Cells in the stratum corneum synthesize a structure termed the cornified envelope (CE) which acts primarily as a barrier to the external environment.

Experiments with transgenic mice suggested that mutations in K14 which collapsed the intracellular filament network were sufficient to cause a mouse blistering disease (2). Human blistering diseases have now been found associated with mutations in this keratin pair. Epidermolysis Bullosa Simplex (EBS) is an autosomal dominant disease of basal cells in which these cells become very fragile, leading to cell lysis and separation of the epidermis from the underlying basement membrane and dermis $(3,4)$. The varying severity of the blistering, resulting in different human skin diseases, is caused by the locations of the mutations in the keratin genes; mutations near the end of the $\alpha$-helical rod domain of the proteins result in the most severe forms of the disease (5). 
Disruption of the suprabasal-specific keratins results in an epidermal blistering disease caused by lysis of suprabasal cells. This disease, Epidermolysis Hyperkeratosis, is characterized by mutations in $\mathrm{Kl}$ or $\mathrm{K} 10$ which lead to a collapse of the network in these layers (6-8). Again, transgenic mice with mutations in the $\mathrm{Kl}$ and $\mathrm{KlO}$ genes have exhibited phenotypes mimicking those of the human diseases (9). Hyperkeratosis and increased cell proliferation are the prominent feature of this human disease. The link between altered cytoskeleton and the increase in proliferation remains to be determined. Other diseases of the suprabasal layers include Epidermolytic Palmoplantar Keratoderma, which results from mutations in the $\mathrm{K} 9$ gene, a keratin specific for the thickened epidermis of the palm and sole (10). Mutations in the keratin 2e gene have recently been found to be responsible for another suprabasal blistering disease, Ichthyosis Bullosa of Siemans $(11,12)$. Almost one half of the keratin mutations are localized to one arginine which is conserved throughout evolution in many intermediate filaments. The association of keratin mutations with human epidermal disease underscores the importance of these filament genes in determining epidermal integrity.

\section{DISEASES OF THE CORNIFIED ENVELOPE}

A major structure produced by keratinocytes in the suprabasal layers is the cornified cell envelope. This highly insoluble envelope is formed by the cross-linking of a number of envelope proteins (via the enzyme transglutaminase) immediately subjacent to the plasma membrane. Gene disruption experiments in mice are currently underway for some of these envelope precursors, and these results should prove most fruitful in determining the contribution of these genes to epidermal disease. An exciting recent development is the association of mutations in the keratinocyte transglutaminase gene with the scaling disease Lamellar Ichthyosis (13).

\section{DEFECTS IN CELL ADHESION: IMMUNOLOGICAL}

Both cell-cell and cell-matrix adhesion are important facets of epidermal homeostasis. Keratinocytes in the basal layer attach to the un- derlying basement membrane through structures termed hemi-desmosomes which contain both the bullous pemphigoid antigen BP-180 (which has a collagen domain) and $\alpha_{6} \beta_{4}$ integrin. In patients with the disease Bullous Pemphigoid, antibodies to components of the hemi-desmosomes are found in the serum (14-16). An animal model for pemphigoid will allow for detailed study of the pathophysiology of this disease.

In patients with the diseases Pemphigus Vulgaris and Pemphigus Folliaceous, autoantibodies to the desmoglein components of desmosomes results in decreased cell-cell adhesion and blister formation (17). It is clear that autoimmune reactions to hemi-desmosomal components can result in human skin disease.

\section{DEFECTS IN CELL ADHESION: GENETIC}

Mutations in the laminin 5 (also known as nicein or kalinin) chain component of the anchoring filaments are found in the recessive blistering disease Junctional Epidermolysis Bullosa. These mutations can result in a complete separation of the epidermis from the dermis at the basement membrane junction (18-19). In two genetic disorders for which the causative gene defect is not yet identified, there is epidermal disruption and loss of epidermal integrity. Darier's Disease, whose gene maps to chromosome 12q23-24 (20), is characterized by blistering, hyperkeratosis, sun sensitivity, and increased apoptosis. A similar disorder called Haley's-Haley's Disease maps to a different chromosomal location and has symmetrical intertriginous blistering often precipitated by cutaneous infection.

Other molecules important in epidermal cell adhesion are the integrins. The integrins are receptors for extracellular matrix proteins and are known to regulate both keratinocyte proliferation and differentiation. Although aberrant patterns of expression of these genes have been observed in psoriasis and squamous cell carcinomas (21-23), a causative role has yet to be established.

\section{OTHER EPIDERMAL DISEASES}

Genetic diseases involving a variety of other aspects of epidermal biology have also been characterized. The cause of the disease, Xeroderma 
Pigmentosum, was found nearly 20 years ago by Cleaver to be a defect in DNA repair. This defect renders the epidermis extremely sensitive to sun damage (24). Genetic mapping studies of Xeroderma Pigmentosum and of Cockayne's Syndrome, another disease caused by a DNA-repair defect, have yielded the locations of genes to correct these diseases. The genes are highly conserved among yeast, Drosophila, and hummans, and much excitement has been recently generated by a link between transcription and DNA repair (25). Recessive $X$-linked Ichthyosis is a milder form of ichthyosis which has been found to be a defect in the steroid sulfatase gene (located on chromosome Xp). The scaly skin phenotype observed in these patients is caused by a disorder of lipid metabolism due to low levels of the enzyme (26).

Although keratinocytes comprise the bulk of the epidermis, other cell types are also present, and diseases associated with these cell types illustrate different molecular and cellular mechanisms which are undoubtedly important. Several diseases affecting skin pigmentation are due to defects in melanocytes. In albinism, although melanocytes are present, there is a defect in the tyrosinase gene and other genes involved in melanin biosynthesis $(27,28)$. Another melanocytic disease, Piedbaldism, is due to the complete absence of melanocytes in the epidermis. The absence of melanocytes from the skin is due, at least in part, to mutations in the c-kit gene (29). This gene is responsible for the migration and proliferation of melanocytes, and may well be involved in stem cell maintenance in other cell types as well. There is also a myriad of immune cells in the epidermis which undoubtedly contribute to epidermal disease. These cell types include antigen-presenting Langerhans cells and $\gamma \delta \mathrm{T}$-cells, but this topic is beyond the scope of the present review.

\section{SUMMARY}

The complex nature of epidermal tissue homeostasis is borne out by the range of diseases affecting this tissue. Indeed, mutations in proteins involved in intracellular integrity and cellcell or cell-matrix adhesion can cause disease in an appropriate epidermal compartment. The most important realization in epidermal disease in the last two years has been that point mutations in key structural genes can result in filaments collapsing, cell cytolysis, or cell adhesion defects; and that these defects can result in severe human skin disease. Now that these associations have been made, the important next step will be to alleviate the suffering of these patients. Animal models will be an important part of these investigations; many molecules including growth factors, oncogenes, and cell adhesion molecules have been targeted to the epidermis of transgenic mice to investigate their role in disease. Such animal models should also elucidate the causes of diseases like psoriasis, a very common skin disease, the molecular basis of which remains elusive.

Gene therapy involving the replacement of defective genes or local delivery of therapeutic molecules will be one of the main goals in alleviating these known epidermal diseases. Such protocols in the epidermis are aided by the relative accessibility of the skin and the presence of the "stem cells" in relatively accessible compartments. Indeed, as the last few years have shed much light on the genetic causes of epidermal disease, it is hoped that the next several years will prove as illuminating in the alleviation of these diseases.

\section{REFERENCES}

1. Albers K and Fuchs E (1987) The expression of mutant epidermal keratin cDNAs transfected in simple epithelial and squamous cell carcinoma lines. J. Cell Biol. 105: 791-806.

2. Vassar R, Coulombe PA, Degenstein L, et al. (1991) Mutant keratin expression in transgenic mice causes marked abnormalities resembling a human genetic skin disease. Cell 64: 365-380.

3. Coulombe PA, Hutton ME, Letai A, et al. (1991) Point mutations in human keratin 14 genes of epidermolysis bullosa simplex patients: Genetic and functional analysis. Cell 66: 1301-1311.

4. Lane EB, Rugg EL, Navsaria H, et al. (1992) A mutation in the conserved helix termination peptide of keratin 5 in hereditary skin blistering. Nature 356: 244-246.

5. Stephens K, Sybert VP, Wijsman EM, et al. (1993) A keratin 14 mutational hot spot for epidermolysis bullosa simplex, DowlingMeara: Implications for diagnosis. J. Invest. Dermatol. 101: 240-243.

6. Cheng J, Snyder AJ, Yu QC, et al. (1992) The genetic basis of epidermolytic hyperkerato- 
sis: A disorder of differentiation-specific epidermal keratin genes. Cell 70: 811-819.

7. Chipev CC, Korge BP, Markova N, et al. (1992) A leucine-proline mutation in the $\mathrm{Hl}$ subdomain of keratin 1 causes epidermolytic hyperkeratosis. Cell 70: 821-828.

8. Rothnagel JA, Dominey AM, Dempsey LD, et al. (1992) Mutations in the rod domains of keratins 1 and 10 in epidermolytic hyperkeratosis. Science 257: 1128-1130.

9. Fuchs E, Esteves RA, and Coulombe PA. (1992) Transgenic mice expressing a mutant keratin 10 gene reveal the likely basis for epidermolytic hyperkeratosis. Proc. Natl. Acad. Sci. U.S.A. 89: 6906-6910.

10. Reis A, Kuster W, Eckardt R, Speling K. (1992) Mapping of a gene for epidermolytic palmoplantar keratoderma to the region of the acidc keratin gene cluster at 17q12-q21. Hum. Genet. 90: 113-116.

11. Steiklen PM, Kremer H, Vakilzadeh F, et al. (1994) Genetic linkage of the keratin type II gene cluster with icthyosis bullosa of Sieman's and with autosomal dominant ichthyosis exfoliativa. J. Invest. Dermatol. 103: 282-285.

12. Kremer H, Zeeuwen $\mathrm{P}$, McLean WHI, et al. (1994) Ichthyosis bullosa of Sieman's is caused by mutations in the keratin $2 \mathrm{e}$ gene. J. Invest. Dermatol. 103: 286-289.

13. Huber $M$, Rettler I, Bernasconi $K$, et al. (1994) Keratinocyte transglutaminase in lamellar ischthyosis. Science (In press).

14. Mutasim DF, Takahashi Y, Labib RS, et al. (1985) A pool of bullos pemphigoid antigen(s) is intracellular and associated with the basal cell cytoskeleton-hemidesmosome complex. J. Invest. Dermatol. 84: 47-53.

15. Westgate GE, Weaver AC, Couchman JR. (1985) Bullous pemphigoid antigen localization suggests an intracellular association with hemidesmosomes. J. Invest. Dermatol. 84: 218-224.

16. Regnier M, Vaigot $P$, Michel S, et al. (1985) Localization of bullous pemphigoid antigen (BPA) in isolated human keratinocytes. $J$. Invest. Dermatol. 85: 187-190.

17. Amagai $M$, Klaus-Kovtun $V$, and Stanley JR. (1991) Autoantibodies against a novel epithelial cadherin in pemphigus vulgaris, a disease of cell adhesion. Cell 67: 869-877.
18. Aberdam D, Galliano M-F, Vailly J, et al. (1994) Herlitz's junctional epidermolysis bullosa is genetically linked to mutations in the gene for the $\gamma 2$ subunit (LAMC2) of nicein/kalinin (laminin 5). Nature Gen. 6: 299-304.

19. Pulkkinen L, Christiano AM, Airenne T, et al. (1994) Mutations in the $\gamma 2$ chain gene (LAMC2) of kalinin/laminin 5 in the junctional forms of epidermlysis bullosa. Nature Gen. 6: 293-298.

20. Ikeda S, Wakem $P$, Haake A, et al. (1994) Localization of the gene for Darier's disease to a 5-cM interval on chromosome 12q. $J$. Invest. Dermatol. 103: 478-481.

21. Ralfkiaer E, Thomsen K, Vejlsgaard GL. (1991) Expression of a cell adhesion protein (VLA- $\beta$ ) in normal and diseased skin. Br. $J$. Dermatol. 124: 527-532.

22. Hertle MD, Kubler MD, Leigh IM, Watt FM. (1992) Aberrant integrin expression during epidermal wound healing and in psoriatic epidermis. J. Clin. Invest. 89: 1892-1901.

23. Tennenbaum $\mathrm{T}$, Weiner $\mathrm{AK}$, Belanger $\mathrm{AJ}$, et al. (1993) The suprabasal expression of $\alpha 6 \beta 4$ integrin is associated with a high risk for malignant progression in mouse skin carcinogenesis. Cancer Res. 53: 4803-4810.

24. Cleaver JE. (1968) Defective repair replication of DNA in xeroderma pigmentosum. Nature 218: 652-656.

25. Weeda G, Wiengant J, van der Ploeg $M$, et al. (1991) Localization of the xeroderma pigmentosum group B-correcting gene ERCC 3 to human chromosome $2 \mathrm{q} 21$. Genomics 10: 1035-1040.

26. Schaeffer L, Roy R, Humbert S, et al. (1993) DNA repair helicase: a component of BTF2 basic transcription factor. Science 260: 58-63.

27. Ramsay M, Colman M-A, Stevens G, et al. (1992) The tyrosine-positive oculocutaneous albinism locus maps to chromosome 15q11.2-q12. Am. J. Hum. Genet. 51: 879884.

28. Tomita Y. (1993) Tyrosine gene mutations causing oculocutaneous albinism. J. Invest. Dermatol. 100: 186S-190S.

29. Rinchik EM, Bultman SJ, Horthemke B, et al. (1993) A gene for the pink-eyed dilution locus and for human type II oculocutaneous albinism. Nature 361: 72-76. 